\title{
COMPARISON OF COMBINED EFFECTS OF PHYSICAL AND MENTAL PRACTICE WITH PHYSICAL PRACTICE ALONE ON FUNCTIONAL INDEPENDENCE IN PARKINSON (PD) PATIENTS
}

\author{
Marya Lokhandwala, Vivek Kulkarni, Manasa Nair.
}

11/12, Thube Park, Shivajinagar, Pune, Maharashtra, India.

\section{ABSTRACT}

Objectives: To compare the effects of combined mental and physical practice with physical practice alone on functional independence in Parkinson disease patients.

Study design: Randomized control trial.

Setting: Tertiary health care center

Participants: 30 subjects were recruited with idiopathic Parkinson's disease, 60-75 years of age, who were between 1.5 -3 on the Hoehn \& Yahr classification \& had a score of 24 on the modified Mini Mental State Examination (MMSE). They were randomly assigned into two groups i.e. the experimental group $(\mathrm{N}=15)$ which received physical practice along with mental imagery \& the control group $(N=15)$ which received physical practice alone.

Outcome measures: Performance time of functional movement tasks such as supine to standing position \& then standing to supine position were measured. In addition to this the Timed Up\& Go (TUG) test and the mental, motor \& ADL subsets of the Unified Parkinson's Disease Rating Scale (UPDRS) \& Stroop test for cognitive assessment were administered.

Results: There was a significant difference in the performance time of supine to standing \& standing to supine position ( $p 0.00$ ), TUG test ( $p 0.003$ ) \& Stroop test errors ( $p$ 0.004). However there was no significant difference in the UPDRS and stroop test time scores.

Conclusion: This study shows that the experimental group performed better during the functional tasks and cognitive Stroop test, however there was no significant improvement in UPDRS scores.

KEY WORDS: Mental practice, functional independence, Parkinson's disease.

Address for correspondence: Dr. Manasa Nair, 1/12, Thube Park, Shivajinagar, Pune, Maharashtra 411005, India. E-Mail: manasanair22@gmail.com

Access this Article online

Quick Response code

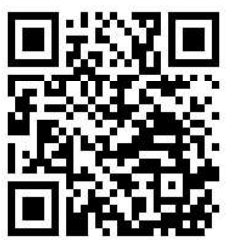

DOI: $10.16965 /$ ijpr.2019.160

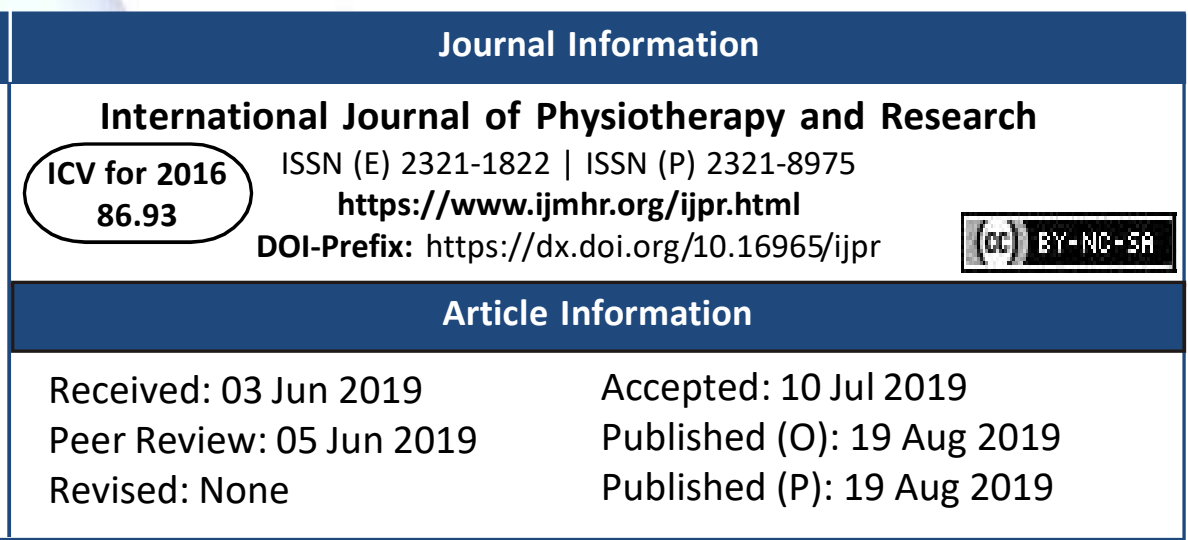

\section{INTRODUCTION}

Parkinson's disease is the second most common, progressive multi-system neurodegenerative disease affecting people mainly in later years of life [1].

In most cases, symptoms start in one side of the body, with contra lateral symptoms appear- ing within a few years. The body posture becomes stooped, there is axial and limb rigidity with or without cogwheel phenomenon, tendency for a shuffling gait and lack of arm swing while walking [2].

The symptoms are generally categorized as motor and non-motor symptoms. There are five 
primary motor symptoms of Parkinson's disease: tremor, rigidity, bradykinesia (slow movement), postural instability (balance problems), and walking/gait problems [3]. The latter three are the most handicapping symptoms of Parkinson's disease leading to difficulties with everyday tasks such as dressing, feeding, bathing and transfers [4].

The earliest non- motor symptoms include problems with executive functioning, i.e., planning and organizing goal-directed behavior [5]. With advancing disease, the non- motor symptoms generally become more troublesome for the patients than themotor symptoms [2].

Physiotherapy intervention for Parkinson's is divided into exercise and movement strategy training. Exercise includes training of strength, endurance, flexibility, balance and functional practice; Movement Strategy Training uses external cueing and attention strategies to improve step length, freezing and turning during walking and in activities of daily living [6]. Mental practice by applying motor imagery is defined as using the imagery of a motor act in an attempt to learn and improve outcome without an overt sensory input-motor output relationship[7-9]. Motor imagery practice is close to the notion of simulation used in cognitive neuroscience. The simulation hypothesis states that thinking consists of simulated interaction with the environment, and rests on the following three core assumptions: (1) Simulation of actions: we can activate motor structures of the brain in a way that resembles activity during a normal action without causing any overt movement; (2) Simulation of perception: imagining perceiving something is essentially the same as actually perceiving it; (3) Anticipation: there exists associative mechanisms that enable both behavioral and perceptual activity to elicit other perceptual activity in the sensory areas of the brain [10]. According to this theory, an activation of motor processes is required for mental simulation.

Motor imagery process retrieves information from Long- term memory to working memory [11]. It causes activation of specific cortical and sub cortical areas of the cerebral cortex which are involved in the early stages of motor control. These cortical motor areas are linked closely to the cerebellum and the basal ganglia, thereby creating feedback loops [12]. Clinically, this revealed that motor images retain many of the properties, in terms of temporal regularities, programming rules and biomechanical constraints, which were observed in the corresponding real action during execution [13].

Mental practice has been used successfully in combination with actual practice to rehabilitate motor deficits in a patient with sub-acute stroke [14]. It also seems to improve balance in individuals with multiple sclerosis and in elderly women [15]. As for patients with PD, mental practice prior to real performance has recently been recommended [8] and further research is required to substantiate how motor imagery actually benefits patients with Parkinson Disease.

\section{METHODOLOGY}

This single blinded randomized control trial was carried out in a tertiary health care setup. All patients with Parkinson disease were screened and those who fulfilled the inclusion criteria were recruited. They were randomly allocated into two groups using a computer generated random allocation table. Both the groups received a total of 20 therapy sessions on 5 days of the week for 4 weeks. The control group received conventional therapy exercises for 1 hour, whereas the experimental group received 45 minutes of conventional therapy along with 15 minutes of mental imagery practice of functional activities. This was done so as to keep the duration of intervention of both the groups same.

\section{Inclusion criteria:}

- Age between 60-75 years

- Idiopathic Parkinson disease between stages 1.5 - 3 according to Hoehn and Yahr's classification.

- Mini Mental State Examination score of greater or equal to 24points.

- Ability to perform Motor imagery as assessed on MIQ- RS scale [16]. 


\section{Exclusion criteria:}

- Parkinson patients with dementia.

- Subjects with co-existing musculoskeletal abnormalities affecting functional outcome e.g. severe back pain, recent fracture which is less than 6 months old.

- Patients suffering from frequent hallucinations, dyskinesias and serious depression.

- Patients with co-existent visual disorder or hearing impairment based on the opinion of doctor in charge.

- Ankle contracture or severe TA tightness.

\section{Outcome Measusres:}

1. Performance time of functional movement task

- Timed up and go (TUG) performance time

- Supine to Stand (St:ST) performance time

- Stand to Supine (ST:tS) performance time

2. Unified Parkinson's Disease Rating Scale(UPDRS)scores

- Mental, Motor, and ADL subsets

3. Cognitive assessment : Stroop Test

- Time of task completion and number of errors

\section{Procedure:}

After approval from the ethical committee was obtained, a randomized controlled trial was performed on Parkinson Patients. Screening was done by intervener according to inclusion and exclusion criteria. Informed written consent was taken from subjects and they were asked not to participate in any other exercise program during the duration of the study.They were then allocated to Control and Experimental groups based on computerized Random Allocation, after which the assessor pre- assessed the subjects on outcome measures of Timed functional sequences, UPDRS scores and Stroop test scores.

\section{Intervention Procedures:}

The exercises given to each subject in both groups were performed at the same duration of time everyday such that patients were in their "ON" state post-medication. The physical exercises given in both groups were similar. "Home assignment" was given to both groups with the same instructions for exercises. The exercises given to experimental group included Imagery practice following guidelines of PETTLEP for motor Imagery. Mental imagery for the functional tasks was taught to patients.

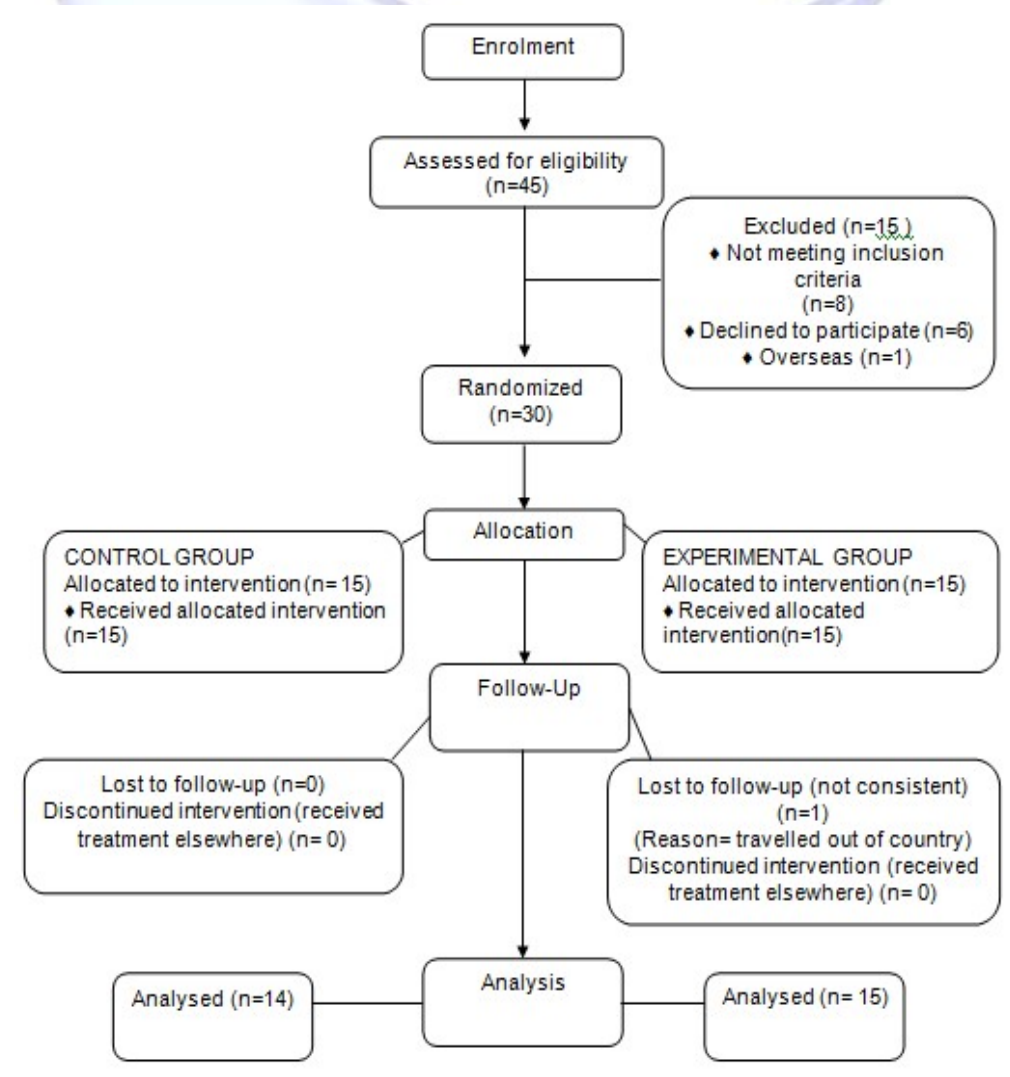


The exercises given in control group: (Table 1)

\begin{tabular}{|c|c|c|}
\hline Relaxation Exercises & Callisthenic Exercises & Functional Activities \\
\hline $\begin{array}{l}\text { Gentle diaphragmatic } \\
\text { breathing exercises }\end{array}$ & Sitting without support and with weights : & $\begin{array}{l}\text { Sit to stand from a standard chair } \\
\text { with holds while descending }\end{array}$ \\
\hline \multirow{3}{*}{$\begin{array}{l}\text { Rhythmic rotations of } \\
\text { trunk and bilateral lower } \\
\text { limbs }\end{array}$} & $\begin{array}{l}\text { Taking Bilateral arms up and back in a } \\
\text { diagonal pattern assisting trunk extension }\end{array}$ & $\begin{array}{l}\text { Retrieving objects from floor and } \\
\text { reaching in different directions }\end{array}$ \\
\hline & $\begin{array}{l}\text { Swinging arms up in opposite directions with } \\
\text { trunkrotations }\end{array}$ & $\begin{array}{l}\text { Walking around and over } \\
\text { obstacles }\end{array}$ \\
\hline & & Backward walking \\
\hline $\begin{array}{l}\text { Jacobson's progressive } \\
\text { relaxation post exercise }\end{array}$ & $\begin{array}{l}\text { In supine: } \\
\text { - Bridging with holds In prone: } \\
\text { - Bhujangasana with holds } \\
\text { In standing: }\end{array}$ & $\begin{array}{l}\text { (Verbal feedback for taking larger } \\
\text { steps was given throughout the } \\
\text { gait activities) }\end{array}$ \\
\hline & - Throwing and catching a ball & \\
\hline
\end{tabular}

The patient first performs movement and is made aware of the wrong movement patterns. The therapist then performs correct movement sequence while patient observes attentively and carefully imagines himself doing the correct movement and feeling the same (patient may be given external support to assist the imagery process). He then tries to replicate the corrected movement sequence, as imagined. The process ends with the subject performing the corrected movement physically. The complex functional sequences were broken down into simple movements for practice. The act of motor imagery was measured using mental chronometry (by stopwatch) and Heart rate measure (polar heart rate monitor) [17]. Motor imagery practice was given for a maximum duration of 5 minutes at a time [18]. All functional sequences were practiced by imagery preceding the real movement.

\section{Statistical analysis:}

Data was analyzed using Statistical Package for Social Sciences (SPSS) version16. Level of significance is set at $p$ value $<0.05$. Within group analysis for parametric data i.e. TUG test and functional performance times were analyzed using paired t-test and non-parametric data i.e.UPDRS was analyzed using Wilcoxon signed rank test. Between group analysis for parametric data was done using unpaired t-test and for non- parametric data analysis was done using Mann-Whitney U test.

\section{RESULTS}

Of the 30 subjects, 1 subject in the control group did not complete the duration of study; thus 29 subjects were analyzed for the study. The average ages of the subjects were $68.4 \pm 4$.

Table 2: Comparison between mean difference of Pre-Post values of control and experimental group.

\begin{tabular}{|c|c|c|c|}
\hline $\begin{array}{c}\text { Outcome } \\
\text { measures }\end{array}$ & Control group & $\begin{array}{c}\text { Experiment } \\
\text { al group }\end{array}$ & P value \\
\hline TUG & $1.35 \pm 1.0$ & $2.6 \pm 1.0$ & $0.003^{*}$ \\
\hline StST & $0.42 \pm 0.6$ & $3.0 \pm 0.5$ & $0.000^{*}$ \\
\hline STtS & $0.64 \pm 0.4$ & $2.86 \pm 0.9$ & $0.000^{*}$ \\
\hline UPDRS & $16.75 \pm 2.5$ & $13.37 \pm 2.7$ & 0.29 \\
\hline $\begin{array}{c}\text { Stroop Test : } \\
\text { errors }\end{array}$ & $0.28 \pm 0.9$ & $1.2 \pm 0.7$ & $0.004^{*}$ \\
\hline Stroop Test: time & $0.85 \pm 1.7$ & $0.4 \pm 1.7$ & 0.484 \\
\hline
\end{tabular}

Graph 1: Comparison of Mean Change Score of TUG between Control and Experimental group showing significant result

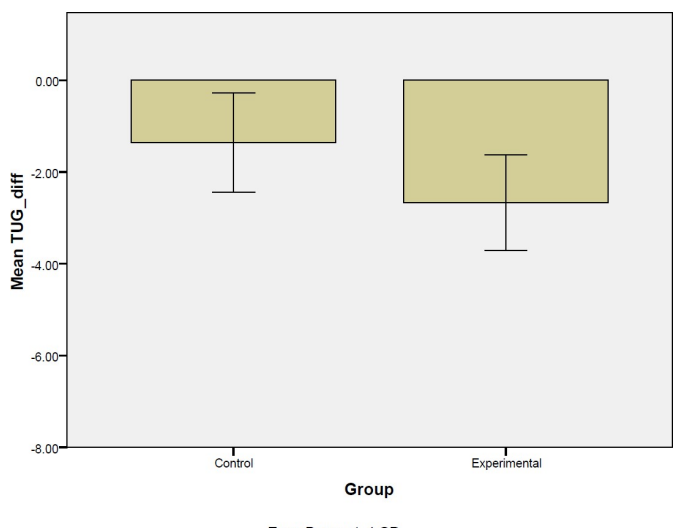


Between group analysis of both groups shows significant results in the mean difference scores of TUG, Supine to stand, Stand to supine time and Stroop test error scores; Whereas UPDRS scores and Stroop test time scores did not show significant difference.

Graph 2: Comparison of Mean Change Score of Supine to stand time between Control and Experimental group showing significant result.

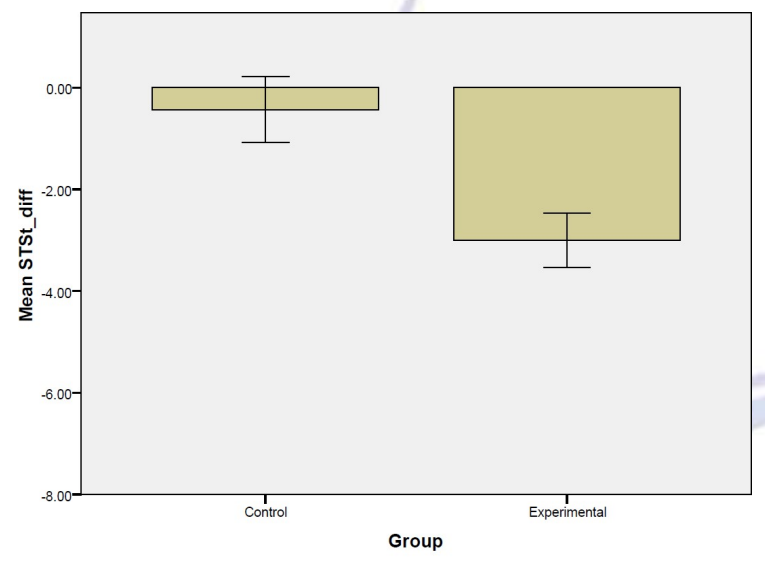

Graph 3: Comparison of mean change score of stand to supine time between control and experimental group showing significant result.

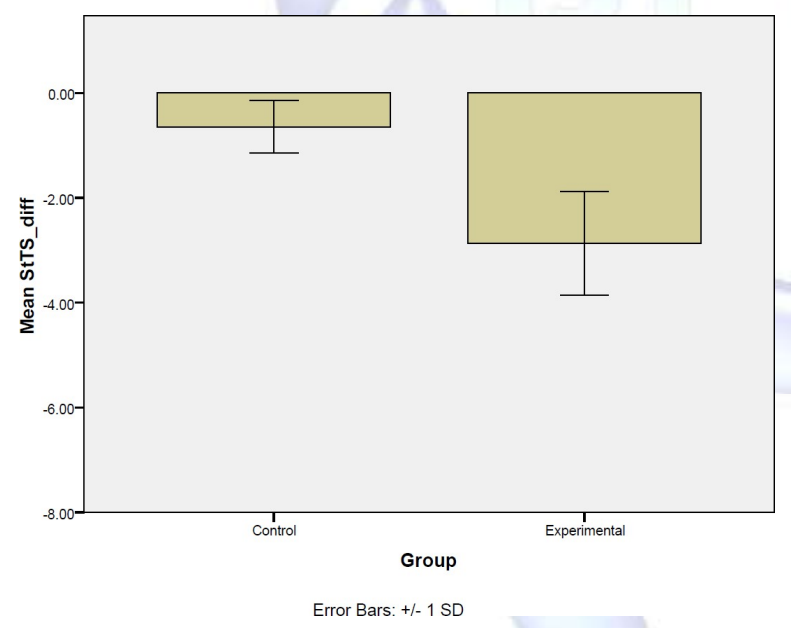

Graph 4: Comparison of mean change score of Stroop errors between control and experimentalgroup showing significant result.

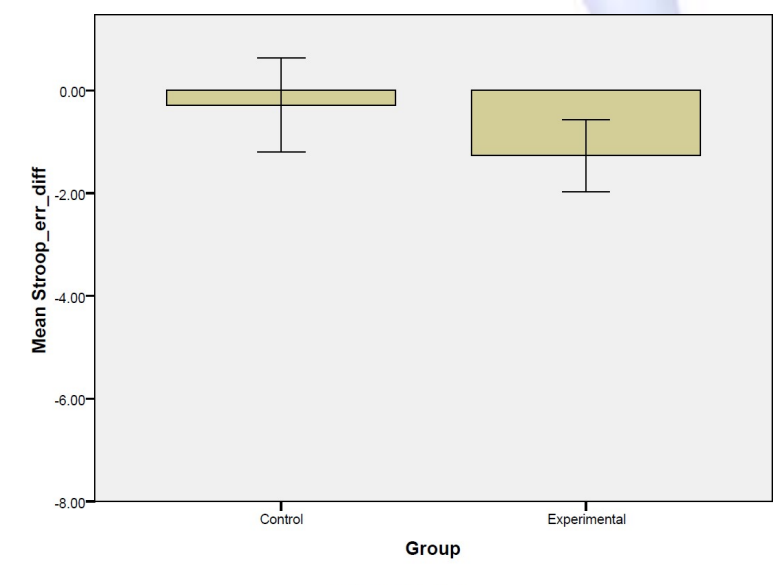

Error Bars: $+/-1 \mathrm{SD}$

\section{DISCUSSION}

The results of this study show us that when physical exercise was integrated with mental practice, a significant change was obtained in the time taken to perform functional tasks, thereby improving the functional status of patients with Parkinson's disease. There was also a reduction in the number of errors during the stroop test; however there was no significant difference in the time taken to perform stroop test.

The higher gains in the experimental group can be attributed to the fact that participants in the experimental group were advised to rehearse the movements via imagery before performing them physically, which therefore resulted in imagery practice multiple times through the day due to ease of application of technique. The experimental subjects reported greater confidence while doing tasks post imagery performance. The notion that imagery practice is beneficial to tasks that have been mastered by the subject in the past makes it more useful for training of functional movements in Parkinson subjects who despite severe motor symptoms, are sometimes still able to perform complex movements almost normally under certain conditions ${ }^{[20]}$ For example, some patients will inexplicably freeze while walking through a doorway, but will have no trouble at all climbing a flight of stairs [21]. The underlying problem has more to do with motor control than with actual motor function.

The basal ganglia [22] is involved in two separate elements of motor control. First, they are involved in providing phasic cues to the supplementary motor area (SMA), which is responsible for activating and deactivating each sub-movement within the movement sequence. Second, they are involved in the transmission of motor set information, i.e., they are responsible for accurate execution of each sub-movement element. Therefore different cueing techniques have been used in physical therapy to enhance basal ganglia function [7]. Motor imagery acts as a form of temporal and spatial cue in which the subject perceive the task and environment relations which helps him to plan better. Experimental group also showed greater gains in the 
mental and motor subsets of the UPDRS scale from the baseline, however there was no significant difference between the scores of the two groups. These improvements in the motor subset of the scale which were seen in both groups and greater in experimental group can be attributed to the fact that both groups were trained in gait tasks, posture improvement and skilled movements requiring postural stability, in addition experimental subjects practiced functional tasks, which could be tailored to their own living environment during practice at home. The ADL subset in either group did not show much improvement.

Although the UPDRS is the gold standard for quantifying response to interventions [23] this scale does not adequately describe the patient's difficulties with physical function and participation and may be less responsive to rehabilitation interventions than to more specific measures of function [19]. The ADL subs core of UPDRS is less affected by fluctuations in motor performance at the time of evaluation and serves better as a predictor of disease progression than quantifying effect of intervention [24].

The experimental group reported increased confidence, arousal and reduced anxiety and depression. These findings are consistent with those mentioned by Pavio in which he states that mental imagery mediates behaviour through either cognitive or motivational mechanisms, which affect specific or general response systems. Task analysis and the functions of memory used in imagery rehearsal may also play a major role to this effect [25].

The Stroop task studies the relations between speed of processing, selective attention capacity and executive functions with working memory and cognitive development in various domains [26]. Stroop test performance time and error scores improved post intervention individually in both groups. On comparison between groups, the experimental group showed better results only for error score whereas the improvement in Stroop performance time was not significantly different than the control group. However, since Stroop test is assessed on the basis of number of errors and amount of time taken which should both be proportional for real improvement; the study demonstrated that as errors decreased, the amount of time increased. This shows that there was no real improvement in cognitive status of subjects belonging to either group.

Motor imagery practice contributes to the development of cognitive strategies beyond its direct positive effects on the practiced tasks .It improves motivation, arousal and concentration as well as attention. It involves mental engagement in spatio- temporal parameters [25]. Despite the positive reasons there was no significant improvement in the test maybe because of the performance anxiety and pressure related to the task; where the subject aims at getting minimum errors in the least performance time possible.

Looking back at literature, Motor imagery is a promising upcoming technique used in neurorehabilitation. It has been used with success in combination with actual practice to rehabilitate motor deficits in stroke and multiple sclerosis. Several studies have shown improvement in skill, strength and function with use of imagery. A recent study by Ruth Tamir used combination of mental imagery with physical practice in treating Parkinson patients and assessed their balance, functional tasks, UPDRS scores, and cognitive abilities. They concluded that the motor imagery and real practice in combination may be more effective in the treatment of Parkinson's Disease especially for reducing bradykinesia $[14,15]$.

These findings are consistent with our study, and compliment the results of this study which proves that combination of mental and physical practice together is a better treatment option than compared to physical practice alone.

\section{CONCLUSION}

This study assesses the effects of a combined protocol of mental and physical practice on functional activities, UPDRS and cognition via Stroop test in Parkinson disease patients. It concludes that combination of mental imagery and physical exercises is more effective than physical exercises alone in reducing bradykinesia during functional activities and reducing fear and anxiety of movement in Parkinson's patients. Therefore combination therapy was more effective than physical exercises alone. 


\section{ABBREVIATIONS}

PD- Parkinson's disease

MMSE- Mini Mental State Examination

TUG-Times Up \& Go

UPDRS- Unified Parkinson's Disease Rating scale

ADL- Activities of daily living

\section{Conflicts of interest: None}

\section{REFERENCES}

[1]. Pringsheim T, Jette N, Frolkis A, Steeves TD. The prevalence of Parkinson's disease: A systematic review and meta analysis. Movement disorders. 2014 Nov;29(13):1583-90.

[2]. Sveinbjornsdottir S. The clinical symptoms of Parkinson's disease. Journal of neurochemistry. 2016 Oct 1;139(S1):318-24.

[3]. Jankovic J. Parkinson's disease: clinical features and diagnosis. Journal of neurology, neurosurgery \& psychiatry. 2008 Apr 1;79(4):368-76.

[4]. Lees, Andrew J.; Hardy, John; Revesz, Tamas (200906-13). "Parkinson'sdisease".

[5]. Williams-Gray $\mathrm{CH}$, Foltynie T, Brayne CE, Robbins TW, Barker RA. Evolution of cognitive dysfunction in an incident Parkinson's disease cohort. Brain. 2007 May29;130(7):1787-98.

[6]. Keus SH, Munneke M, Graziano M, Paltamaa J, Pelosin E, Domingos J, Brühlmann S, Ramaswamy B, Prins J, Struiksma C, Rochester L. European physiotherapy guideline for Parkinson's disease. Royal Dutch society for physical therapy (KNGF) and ParkinsonNet. 2014 Dec.

[7]. Marchese R, Diverio M, Zucchi F, Lentino C, Abbru zzese $\mathrm{G}$. The role of sensory cues in the rehabilitation of parkinsonian patients: a comparison of two physical therapy protocols. Movement Disorders. 2000 Sep1;15(5):879-83.

[8]. Morris ME. Movement disorders in people with Parkinson disease: a model for physical therapy. Physical therapy. 2000 Jun1;80(6):578-97.

[9]. DenisM.Visualimagery and theuse of mental practice inthedevelop mentofmotorskills. Canadian journal of applied sport sciences. Journal canadien des sciences appliquees au sport. 1985 Dec;10(4):4S-16S.

[10]. Munzert J, Lorey B, Zentgraf K. Cognitive motor processes: the role of motor imagery in the study of motor representations. Brain research reviews. 2009 May31;60(2):306-26.

[11]. FarahMJ.Theneurological basis of mentalim-agery: Acomponentialanalysis. Cognition.1984 Dec 31;18(1):245-72.

[12]. Dalla-Corte A, das Neves CM, Anés M, Portuguez MW, Dacosta JC. The Effect of Handedness on Supplementary Motor Area Activation during Complex Motor Tasks. Journal of Behavioral and Brain Science. 2015 Sep10;5(10):458.
[13]. Decety J,Jeannerod M,Prablanc C.The timing of mentally represented actions. Behavioural brain research. 1989 Aug 1;34(1):35-42.

[14]. Page SJ, Levine P, Sisto SA, Johnston MV. Mental practice combined with physical practice for upper-limb motor deficit in subacute stroke. Physical Therapy. 2001 Aug 1;81(8):1455-62

[15]. Fansler CL, Poff CL, Shepard KF. Effects of mental practice on balance in elderly women. Physical Therapy. 1985 Sep 1; 65(9):1332-8.

[16]. Gregg M, Hall C, Butler A. The MIQ-RS: a suitable option for examining movement imagery ability. Evidence-Based Complementary and Alternative Medicine. 2010;7(2):249-57.

[17]. Dickstein R, Deutsch JE. Motor imagery in physical therapist practice. Physical therapy. 2007 Jul 1;87(7):942-53.

[18]. Twining WE. Mental practice and physical practice in learning a motor skill. Research Quarterly. American Association for Health, Physical Education and Recreation. 1949 Dec 1;20(4):432-5.

[19]. Schenkman M, Ellis T, Christiansen C, Barón AE, Tickle-Degnen L, Hall DA, Wagenaar R. Profile of functional limitations and task performance among people with early-and middlestage Parkinson disease. Physical therapy. 2011 Sep 1;91(9):1339-54.

[20]. Glickstein M, Stein J. Paradoxical movement in Parkinson's disease. Trends in neurosciences. 1991 Nov 30;14(11):480-2

[21]. Giladi N, McDermott MP, Fahn S, Przedborski S, Jankovic J, Stern M, Tanner C, Parkinson Study Group. Freezing of gait in PD Prospective assessment in the DATATOP cohort. Neurology. 2001 Jun 26;56(12):1712-21.

[22]. Morris ME, lansek R, Matyas TA, Summers JJ. Stride length regulation in Parkinson's disease: normalization strategies and underlying mechanisms. Brain. 1996 Apr 1;119(2):551- 68.

[23]. Fahn SE. Unified Parkinson's disease rating scale. Recent developments in Parkinson's disease. 1987;2:153

[24]. Harrison MB, Wylie SA, Frysinger RC, Patrie JT, Huss DS, Currie LJ, Wooten GF. UPDRS activity of daily living score as a marker of Parkinson's disease progression. Movement disorders. 2009 Jan 30;24(2):224-30.

[25]. Paivio A. Cognitive and motivational functions of imagery in human performance. Canadian journal of applied sport sciences. Journal canadien des sciences appliquées au sport. 1985 Dec;10(4):22S$8 \mathrm{~S}$.

[26]. Moench L. The Development Of Mental Processing Efficiency Working Memory And Thinking. Development. 2016 Aug 11;1:2.

How to cite this article: Marya Lokhandwala, Vivek Kulkarni, Manasa Nair. COMPARISON OF COMBINED EFFECTS OF PHYSICAL AND MENTAL PRACTICE WITH PHYSICAL PRACTICE ALONE ON FUNCTIONAL INDEPENDENCE IN PARKINSON (PD) PATIENTS. Int J Physiother Res 2019;7(4):3207-3213. DOI: 10.16965/ijpr.2019.160 